\title{
Effects of Shell Encapsulation on the Nonlinear Dynamics of a Microbubble Contrast Agent subjected to Acoustic Energy
}

\author{
Fatimah Dzaharudin ${ }^{1, *}$, Najihah Mohd Ali ${ }^{1}$, Erny Afiza Alias ${ }^{1}$ and Ummu Kulthum \\ Jamaludin $^{1}$ \\ ${ }^{1}$ Faculty of Mechanical Engineering, Universiti Malaysia Pahang, Pekan 26600, Pahang, Malaysia
}

\begin{abstract}
The effects of encapsulating a microbubble on the energy in oscillation and acoustic signature is investigated by solving the governing equations of motion. Numerical data obtained by solving these equations is used to explore the effects of shell on the harmonics of the bubble system. By encapsulating the microbubble with a shell, the bubble will be stiffer resulting in lower amplitude oscillations and an increase in the harmonics of the bubble system upon subjected to acoustic energy. This is desirable in triggering vascular permeability for drug/gene control and release in biomedical applications.
\end{abstract}

\section{Introduction}

Microbubbles were first introduced as ultrasound contrast agents. These bubbles have a typical diameter similar to the size of a red blood cell of less than $10 \mu \mathrm{m}$ thereby allowing similar rheology in microvessels and capillaries throughout the body [1]. Early microbubbles contained gas and were highly soluble in liquid. Upon applying acoustic energy microbubbles undergo volumetric oscillation thus increasing sound scatter and acoustic contrast between blood and surrounding tissues, thereby improving the quality of ultrasonic images [2-3]. The gas in the microbubbles will then dissolve into the blood under the influence of blood pressure and acoustic pressure. The major disadvantage of these gas bubbles, however, is the rapid disappearance due to their high solubility.

Microbubbles were shown to have potential to redraw the boundary of. In addition, since the gas core expands and contracts when exposed to ultrasonic wave, microbubbles have demonstrated various phenomena ranging from subtle effects such as acoustic radiation to highly energetic effects such as inertial cavitation [4-5] These phenomenon may be manipulated and utilised for targeting and controlled release of local drug/gene delivery from microbubbles [6], imaging[7] and enhancement of vascular permeability [8]. In the application of targeted therapy, microbubbles can provide simultaneously diagnostic imaging, drug and gene carrying and delivering capacity with ultrasound as the trigger [9]. Pre-clinical studies of microbubbles have also shown that upon sufficient acoustic energy, the oscillation of microbubbles is able to mediate localised biological effects in tissue such

\footnotetext{
*Corresponding author: fatimahd@ump.edu.my
} 
as increased thermal deposition of enhancement of membrane permeability by focusing the mechanical energy to microscale events distributed throughout the insonified vasculature [4].

One of the important improvements in microbubble design to make this technology clinically relevant is to stabilise the microbubbles and avoid fast dissolution. To do this, microbubbles were enclosed in either a surfactant or a solid shell of albumin [10]. Table 1 summarises characteristics of selected ultrasound contrast agents that are commercially available in the market.

Table 1. Properties of commercially available [9-10]

\begin{tabular}{|c|c|c|}
\hline Contrast Agents & Encapsulation Material & Filling Gas \\
\hline SonoVue ${ }^{\circledR}$ & Phospholipid & Sulphur hexafluoride \\
\hline Sonazoid $^{\mathrm{TM}}$ & Phospholipid & Perfluorocarbon gas \\
\hline Optison $^{\mathrm{TM}}$ & Cross-linked serum albumin & Octafluoropropane \\
\hline Albunex $^{\circledR}$ & Sonicated serum albumin & Air \\
\hline Quantison $^{\mathrm{TM}}$ & Spray-dried serum albumin & Air \\
\hline Albunex & Cross-linked serum albumin & Air \\
\hline
\end{tabular}

Despite the substantial amount of theoretical and experimental research over the years, the contribution of the shell to the dynamics of microbubbles is yet to be fully understood due to their inherent complex nonlinear nature. For example, a study by [11] has associated the collapse of a microbubble and micro jet with the small holes produced within a cell membrane. While this is desirable for drug/gene delivery, if not properly controlled, may cause tissue damage.

Thus, it is of paramount importance to identify instances and parameters that may trigger unwanted inertial cavitation to avoid premature drug release. To optimize and control bubblemediated application and achieve desirable effects, it is necessary to understand and predict bubble dynamics. One aspect of this is the theoretical description of encapsulated microbubbles be understood since it is the shell material that determines many of the functional properties of the microbubble contrast agents.

Here, we study the various effects of shell encapsulation on the microbubble dynamics such as the harmonics of an ultrasound contrast agent based on the shell modelling of a SonoVue ${ }^{\circledR}$ microbubble in an ultrasonic field.

\section{Mathematical Modelling}

\subsection{Free gas bubble oscillation}

A Rayleigh-Plesset [12] equation governing the radial motion of a free microbubble will is given by,

$$
\rho R \ddot{R}+\frac{3}{2} \rho \dot{R^{2}}=P_{0}\left(\frac{R_{0}}{R}\right)^{3 \gamma}-4 \eta_{L} \frac{\dot{R}}{R}-P_{0}-P_{d r v}(t)
$$

where $R(t), R_{0}, \rho, \gamma, \eta_{L}, P_{0}$ and $P_{d r v}$ represent the instantaneous bubble radius, equilibrium bubble radius, density of liquid, polytropic exponent for bubble gas, effective liquid viscosity which accounts for thermal damping, atmospheric pressure and acoustic driving force. The 
gas and liquid parameter values are given by $\rho=1000 \mathrm{~kg} \mathrm{~m}^{-3}, \gamma=1.07, \eta_{L}=2 \times$ $10^{-3} \mathrm{~Pa}$. $\mathrm{S}$ and $P_{0}=101.3 \mathrm{kPa}$ for bubbles in water at $20^{\circ} \mathrm{C}$ [13].

The classical Rayleigh-Plesset equation [12] is one of the most widely used in the study of radial bubble oscillations. However, one major disadvantage of this model that it assumes infinite speed of sound in liquid. This becomes a problem if a bubble spans a significant fraction of the wavelength of sound. This model also neglects gas disassociation and effects of heat and mass transfer which are be significant at large oscillation amplitudes. While there has been many studies that propose more complex mathematical models (see Vokurka et al. [14] for detailed analysis on advantages and disadvantages of the models), here we will assume the case where the liquid sound speed is negligible and oscillation amplitudes are not extreme, thus the classical Rayleigh-Plesset equation, Eq. (1) is a reasonable starting point.

\subsection{Free gas bubble oscillation with shell encapsulation}

A bubble encapsulated by a thin shell is depicted in Fig. 1. Here, an air bubble is enclosed in an incompressible, solid, visco-elastic shell described by a shear viscosity $\eta_{s}$ and shear modulus $G_{s}$ which stabilizes the bubble against dissolution. It is assumed that the liquid surrounding the bubble is incompressible and Newtonian with shear velocity $\eta_{L}$. The surface tension is neglected by assuming that the shell reduces surface tensions at the shell-gas and shell-interfaces to zero.

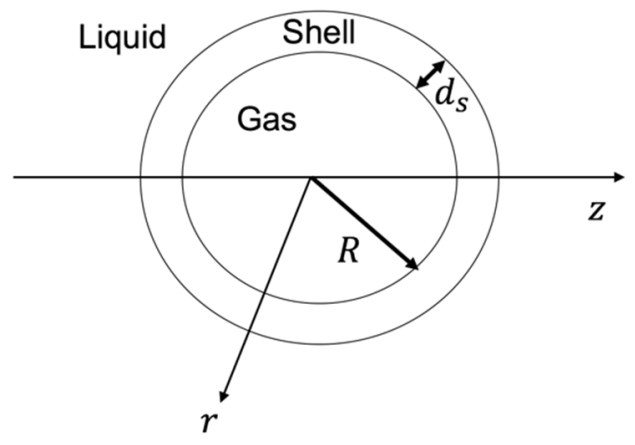

Fig. 1. Schematic sketch of an encapsulated bubble

From these set of assumptions, Hoff et al. [15] derived the following equation of motion for a gas bubble with shell encapsulation,

$$
\begin{aligned}
\rho R \ddot{R}+\frac{3}{2} \rho \dot{R^{2}}= & P_{0}\left(\frac{R_{0}}{R}\right)^{3 \gamma}-4 \eta_{L} \frac{\dot{R}}{R}-12 \eta_{s} \frac{d_{s} R_{0}^{2}}{R^{3}} \frac{\dot{R}}{R}-12 G_{s} \frac{d_{s} R_{0}^{2}}{R^{3}}\left(1-\frac{R_{0}}{R}\right) \\
& -P_{0}-P_{d r v}(t) .
\end{aligned}
$$

The parameters for shell encapsulation is given by shell shear viscosity, $\eta_{s}$ and shell shear modulus, $G_{s}$. In this paper, the values for the shell encapsulation parameters which will be used are $d_{s}=4 \mathrm{~nm}, \eta_{s}=0.5 \mathrm{Pas}$ and $G_{s}=23 \mathrm{MPa}$ [15].

\subsection{Linearisation}

For small pressure amplitudes, the oscillation is linear. Therefore, Eq. (2) may be linearised by setting 


$$
R(t)=R_{0}(1+\epsilon(t)), \text { where }|\epsilon(t)| \leq 1
$$

and substituting into Eq. (2). By ignoring higher order terms and keeping the terms in the first order in $\epsilon$, the forces acting on the surface of the bubble is given by the following linear equation,

$$
\ddot{\epsilon}\left(\rho R_{0}^{2}\right)+\dot{\epsilon}\left(3 \kappa P_{0}+12 G_{s} \frac{d_{s}}{R_{0}}\right)+\epsilon\left(4 \eta_{L}+12 \eta_{s} \frac{d_{s}}{R_{0}}\right)=-P_{d r v}(t) .
$$

By translating Eq. (4) into the frequency domain, the Fourier transformation, we are then able to obtain the linear resonance frequency $f_{0}$ of the shell encapsulated bubble which is given by,

$$
f_{0}=\frac{1}{2 \pi R_{0}} \sqrt{\frac{1}{\rho}\left(3 \kappa P_{0}+12 G_{s} \frac{d_{s}}{R_{0}}\right)}
$$

From Eq. (5), it can be estimated that, compared to the free bubble, the shell resonance frequency increases due to the increase in the stiffness of the bubble through the shear modulus $G_{s}$. Without the shell encapsulation, $d_{s}=0$, and under adiabatic conditions, $\kappa=\gamma$, where $\gamma$ is the polytropic coefficient. Eq. (1) reduces to the well-known Minnaert resonance frequency [16] for gas bubbles without shell encapsulation.

\section{Results}

Here we consider the bubble oscillation for a sinusoidal ultrasound field, $P_{d r v}(t)=$ $\alpha \sin \left(2 \pi f_{\text {ext }} t\right)$, where $\alpha$ and $f_{\text {ext }}$ are the amplitude and frequency of the external field respectively. The study will consider the application of $\alpha=500 \mathrm{kPa}, R_{0}=1.7 \mu \mathrm{m}$ typically used in clinical trials $\backslash$ cite $\{$ Qin2009\} and two different timescales, the period of the forced oscillations $T_{0}=1 / f_{0}$ and the period of the linearized equations due to the natural frequency of the bubble system $T_{\text {ext }}=1 / f_{\text {ext }}$. The initial conditions used by simulations to produce the results in this section is given by $R(t=0)=R_{0}$ and $\dot{R}(t=0)=0$. Fig. 2 shows the response curve of a single microbubble for a gas bubble without any encapsulation (top row) and a SonoVue ${ }^{\circledR}$ microbubble (bottom row). The radius of the bubble are seen to undergo an initial transient phase prior to achieving steady-state at approximately $t / T>5$, where $T=$ $\max \left(T_{0}, T_{\text {ext }}\right)$.

Upon insonation, the microbubble with no shell encapsulation shown in Fig. 2(a) shows a more chaotic behaviour, which is supported by the seemingly random spikes in the Fourier Transform in Fig. 2(b). The shell encapsulating the SonoVue ${ }^{\circledR}$ microbubble in Fig. 2(c), however, exhibits a more consistent oscillation with energy occurring at discrete frequencies displayed by two spikes occurring for every integer multiples of the driving frequency in Fig. 2(d). 

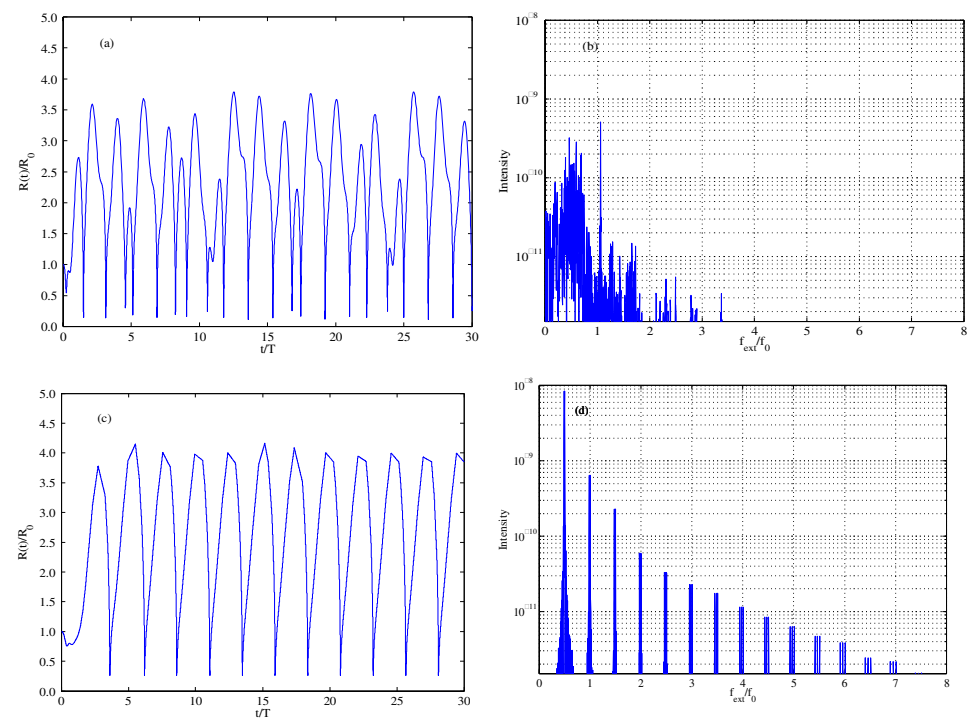

Fig. 2. Left column (a) and (c) shows the normalised radial response and the right column (b) and (d) shows response in the frequency domain for a gas bubble with no encapsulation (top row) and a Sonovue ${ }^{\circledR}$ microbubble (bottom row) for $R_{0}=$ $1.7 \mu \mathrm{m}$ insonated by a sinusoidal pressure wave of frequency, $f_{\text {ext }}=1.2 \mathrm{MHz}$ and pressure amplitude, $\alpha=500 \mathrm{kPa}$.

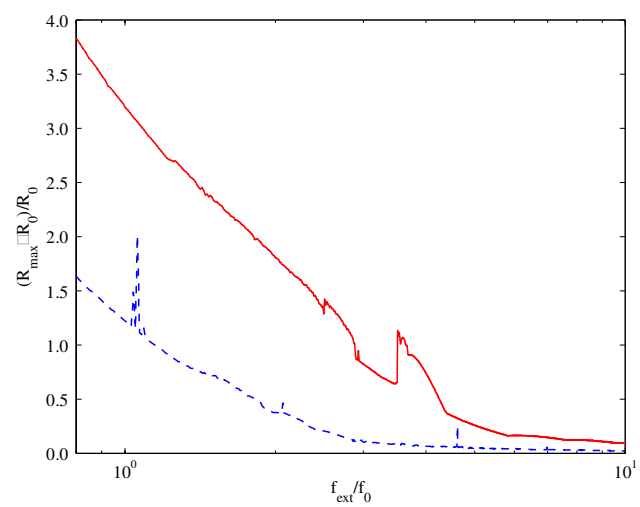

Fig. 3. Frequency response curves for a gas microbubble with no shell encapsulation (red line) and a SonoVue ${ }^{\circledR}$ encapsulated microbubble (dashed blue line) of size $R_{0}=1.7 \mu \mathrm{m}$ subjected to external ultrasound pressure amplitude, $\alpha=500 \mathrm{kPa}$.

This suggest that the shell encapsulation causes the microbubble to be stiffer thus contributing to the more orderly oscillation that the initial chaotic response without the shell. The plot of $\left.\left(\mathrm{R}_{\max }-\mathrm{R}_{0}\right) / \mathrm{R}_{0}\right)$ versus $\mathrm{f}_{\mathrm{ext}} / \mathrm{f}_{0}$ is shown in Fig. 3 where $\mathrm{R}_{\max }$ is the maximum radius of the bubble during it's steady state. Data samples were taken from $250<\mathrm{T} / \mathrm{t}<300$ to ensure that there are no transient oscillations. The peaks observed are the harmonic frequencies, peaks at the multiples of the main resonance of the bubble system. Harmonics have been associated with vascular permeability in clinical studies [8].

While there are more harmonic peaks for the SonoVue ${ }^{\circledR}$ microbubble, the microbubble without encapsulation oscillates at larger oscillation amplitudes, implying weaker stiffness. 
This is supported by the oscillations observed in Fig. 2(a). Microbubbles with no shell encapsulation exhibits a higher larger amplitude oscillation than Sonovue ${ }^{\circledR}$ of the same size. In addition, the absence of the shell shifts the resonance to higher frequency values.

\section{Conclusion}

The governing equations for an encapsulated microbubble and a microbubble with no shell encapsulation was solved. The results show that the shell encapsulating the microbubble will cause the bubble to be stiffer, resulting in lower amplitude oscillations and increasing the harmonics of the bubble system, which is desirable in triggering vascular permeability.

This research was supported by Ministry of Education, Malaysia (FRGS/1/2017/TK03/UMP/03/1) and Universiti Malaysia Pahang, Malaysia (RDU160398 and RDU1703155)

\section{References}

1. J. R. Lindner, J. Song, A. R. Jayaweera, J. Sklenar, S. Kaul, J. Am. Soc. Echocardiogr. 15, 396-403 (2002)

2. H. Becher and P. N. Burns, Handbook of Contrast Echocardiography: Left Ventricular Function and Myocardial Perfusion (Springer Verlag, New York 2000)

3. B. B. Goldberg, J. S. Raichlen, and F. Forsberg, Ultrasound Contrast Agents: Basic Principles and Clinical Applications (Martin Dunitz, London, 2001).

4. K. Ferrara, R. Pollard, M. Borden, Annu. Rev. Biomed. Eng. 9, 415-47 (2007)

5. A. Goyal, F. T. H. Yu, M. G. Tenwalde, X. Chen, A. Althouse, F. S. Villanueva, J. J. Pacella, Ultrasound Med. Biol. 43, 1391-1400 (2017)

6. C. Huang, H. Zhang, R. Bai, Acta Pharmaceutica Sinica B., 7, 447-452 (2017)

7. F. Vignon, W. T. Shi, J. E. Powers, E. C. Everbach, J. Liu, S. Gao, F. Xie, T. R. Porter, IEEE Trans. Ultrason. Ferroelectr. Freq. Control. 4 661-670 (2013)

8. S.-Y. Wu, C. S. Sanchez, G. Samiotaki, A. Buch, V. P. Ferrera, E. E. Konofagou, Sci. Rep. 6, 1-13 (2016)

9. S. Sirsi and M. Borden, Bubble Sci. Eng. Technol. 1, 3-17 (2009)

10. E. Stride, N. Saffari N., Proc Inst Mech Eng H. 6, 429-47 (2003)

11. S. M. Nejad, H. Hosseini, H. Akiyama, K. Tachibana. Theranostics, 6, 446-455 (2016)

12. M. S. Plesset, ASME J. Appl. Mech. 16, 277-282 (1949)

13. J. Tu, J. Guan, Y. Qiu, T. J. Matula, J. Acoust. Soc. Am. 6, 2954-62 (2009)

14. K. Vokurka. Acustica. 59, 214-219 (1986)

15. L. Hoff, Acoustic Characterization of Contrast Agents for Medical Ultrasound Imaging (Kluwer Academic Publishers, London 2001)

16. M. Minnaert, Philos. Mag. 16, 235-248 (1933) 